\title{
Higher-order Euler-type polynomials and their applications
}

Aykut Ahmet Aygunes*

\section{"Correspondence:}

aygunes@akdeniz.edu.tr

Department of Mathematics,

Faculty of Science, University of

Akdeniz, Antalya, TR-07058, Turkey

\begin{abstract}
In this paper, we construct generating functions for higher-order Euler-type polynomials and numbers. By using the generating functions, we obtain functional equations related to a generalized partial Hecke operator and Euler-type polynomials and numbers. A special case of higher-order Euler-type polynomials is eigenfunctions for the generalized partial Hecke operators. Moreover, we give not only some properties, but also applications for these polynomials and numbers.
\end{abstract}

AMS Subject Classification: 08A40; 11F25; 11F60; 11B68; 30D05

Keywords: generalized partial Hecke operators; higher-order Euler-type polynomials; higher-order Euler-type numbers; Apostol-Bernoulli polynomials; Frobenius-Euler polynomials; Euler polynomials; Euler numbers; functional equation; generating functions

\section{Introduction}

In this section, we define generalized partial Hecke operators and we give some notation for these operators. Also, we define generalized Euler-type polynomials, ApostolBernoulli polynomials and Frobenius-Euler polynomials.

Throughout this paper, we use the following notations:

$\mathbb{N}=\{1,2, \ldots\}, \mathbb{N}_{0}=\{0,1,2, \ldots\}=\mathbb{N} \cup\{0\}$. Also, as usual, $\mathbb{Z}$ denotes the set of integers, $\mathbb{R}$ denotes the set of real numbers and $\mathbb{C}$ denotes the set of complex numbers. We assume that $\ln (z)$ denotes the principal branch of the multi-valued function $\ln (z)$ with an imaginary part $\Im(\ln (z))$ constrained by $-\pi<\Im(\ln (z)) \leq \pi$. Furthermore, $0^{n}=1$ if $n=0$, and $0^{n}=0$ if $n \in \mathbb{N}$.

$$
N(M)=\left(N_{1}, N_{2}, \ldots, N_{M}\right),
$$

where $M \in \mathbb{N}$ and $N_{1}, N_{2}, \ldots, N_{M} \in \mathbb{N}$.

Let $a \in \mathbb{N}$ and $\chi_{a, N(M)}$ be a function depending on $a, N_{1}, N_{2}, \ldots, N_{M}$ such that

$$
\chi_{a, N(M)}: \mathbb{N}_{0} \rightarrow \mathbb{C}
$$

$\chi_{a, N(M)}$ is defined by

$$
\chi_{a, N(M)}(k)=\prod_{j=1}^{M} \xi^{k}\left(N_{j}\right),
$$

@ 2013 Aygunes; licensee Springer. This is an Open Access article distributed under the terms of the Creative Commons Attribution License (http://creativecommons.org/licenses/by/2.0), which permits unrestricted use, distribution, and reproduction in any medium, provided the original work is properly cited. 
where $0 \leq k \leq a-1, j \in\{1,2, \ldots, M\}$ and

$$
\xi\left(N_{j}\right)=e^{\frac{2 \pi i}{N_{j}}} .
$$

$\chi_{a, N(M)}$ satisfies the following properties:

(i) $\chi_{a, N(M)}$ is a periodic function with $N_{1} N_{2} \cdots N_{M}$.

(ii) If we take $N_{1} \geq 2$ and $N_{2}=N_{3}=\cdots=N_{M}=1$, we have

$$
\chi_{a,\left(N_{1}, 1,1, \ldots, 1\right)}(k)=\xi^{k}\left(N_{1}\right) \xi^{k}(1) \xi^{k}(1) \cdots \xi^{k}(1)=\xi^{k}\left(N_{1}\right) .
$$

We note that replacing $N(M)$ by $\left(N_{1}, 1,1, \ldots, 1\right), \chi_{a, N(M)}$ is reduced to $\xi^{k}\left(N_{1}\right)(c f$. [1]).

Let $\mathbb{C}[x]$ be a ring of polynomials with complex coefficients. By using $\chi_{a, N(M)}$, we give the following definition.

Definition 1.1 [2] Let $P \in \mathbb{C}[x]$. The generalized partial Hecke operator of $T_{\chi_{a, N(M)}}$ is defined by

$$
T_{\chi_{a, N(M)}}(P(x))=\sum_{k=0}^{a-1} \chi_{a, N(M)}(k) P\left(\frac{x+k}{a}\right) .
$$

The operator $T_{\chi_{a, N(M)}}$ satisfies the following properties:

(i) $T_{\chi a, N(M)}$ is linear on $\mathbb{C}[x]$ and

$$
T_{\chi_{a, N(M)}}: \mathbb{C}[x] \rightarrow \mathbb{C}[x]
$$

(ii) $T_{\chi_{a, N(M)}}$ preserves the degree of the polynomials on $\mathbb{C}[x]$.

(iii) If we take $N_{1} \geq 2$ and $N_{2}=N_{3}=\cdots=N_{M}=1$, we have

$$
T_{\chi_{a, N_{1}}}(P(x))=\sum_{k=0}^{a-1} \xi^{k}\left(N_{1}\right) P\left(\frac{x+k}{a}\right)
$$

Remark 1.2 Setting $N(M)=\left(N_{1}, 1,1, \ldots, 1\right), T_{\chi_{a,\left(N_{1}, 1,1, \ldots, 1\right)}}$ is reduced to $T_{\chi_{a, N_{1}}}(c f .[1])$.

The generating function of generalized Euler-type numbers $P_{n, N(M)}$ is given by

$$
\mathcal{F}_{N(M)}(t)=\sum_{n=0}^{\infty} P_{n, N(M)} \frac{t^{n}}{n !}=\frac{\prod_{j=1}^{M} \xi\left(N_{j}\right)-1}{-1+e^{t} \prod_{j=1}^{M} \xi\left(N_{j}\right)}
$$

[2].

Now, we give the definition of Euler-type polynomials as follows.

Definition 1.3 [2] The polynomial $P_{n, N(M)}$ is defined by means of the following generating function:

$$
\mathcal{F}_{N(M)}(t, x)=\sum_{n=0}^{\infty} P_{n, N(M)}(x) \frac{t^{n}}{n !}=\frac{\left(\left(\prod_{j=1}^{M} \xi\left(N_{j}\right)\right)-1\right) e^{t x}}{\left(\prod_{j=1}^{M} \xi\left(N_{j}\right)\right) e^{t}-1}
$$


where

$$
\left|t+\sum_{j=1}^{M} \frac{2 \pi i}{N_{j}}\right|<2 \pi .
$$

The polynomial $P_{n, N(M)}$ satisfies the following properties:

(i) $P_{n, N(M)} \in \mathbb{C}[x]$.

(ii) $P_{n, N(M)}$ is a polynomial with degree $n$ and depends on $N_{1}, N_{2}, \ldots, N_{M}$.

(iii) If we take $N_{1} \geq 2$ and $N_{2}=N_{3}=\cdots=N_{M}=1$, we have

$$
\sum_{n=0}^{\infty} P_{n, N_{1}}(x) \frac{t^{n}}{n !}=\frac{\left(\xi_{N_{1}}-1\right) e^{t x}}{\xi_{N_{1}} e^{t}-1}
$$

where

$$
\left|t+\frac{2 \pi i}{N_{1}}\right|<2 \pi
$$

(iv) We derive the following functional equation:

$$
\mathcal{F}_{N(M)}(t, x)=\mathcal{F}_{N(M)}(t) e^{t x},
$$

so that, obviously,

$$
P_{n, N(M)}(0)=P_{n, N(M)}
$$

We now are ready to define Euler-type numbers and polynomials with order $k$.

Definition 1.4 Euler-type numbers with order $k, P_{n, N(M)}^{(k)}$, are defined by means of the following generating functions:

$$
\mathcal{F}_{N(M)}^{(k)}(t)=\sum_{n=0}^{\infty} P_{n, N(M)}^{(k)} \frac{t^{n}}{n !}
$$

where $k \in \mathbb{N}$ and

$$
\left|t+\sum_{j=1}^{M} \frac{2 \pi i}{N_{j}}\right|<2 \pi .
$$

Euler-type polynomials with order $k$ are given by the following functional equation:

$$
\mathcal{F}_{N(M)}^{(k)}(t, x)=\mathcal{F}_{N(M)}^{(k)}(t) e^{t x}=\sum_{n=0}^{\infty} P_{n, N(M)}^{(k)}(x) \frac{t^{n}}{n !}
$$

We see that

$$
\mathcal{F}_{N(M)}^{(0)}(t, x)=e^{t x} .
$$


Thus we obtain

$$
P_{n, N(M)}^{(0)}(x)=x^{n}
$$

Remark 1.5 Substituting $k=1$ into (4), we get (2). Therefore, (3) reduces to (1); that is,

$$
P_{n, N(M)}^{(1)}(x)=P_{n, N(M)}(x)
$$

so that, obviously,

$$
P_{n, N(M)}^{(1)}(0)=P_{n, N(M)}
$$

By using (4) and (3), we obtain

$$
\sum_{n=0}^{\infty} P_{n, N(M)}^{(k)}(x) \frac{t^{n}}{n !}=\sum_{n=0}^{\infty} P_{n, N(M)}^{(k)} \frac{t^{n}}{n !} \sum_{n=0}^{\infty} x^{n} \frac{t^{n}}{n !} .
$$

Therefore, we get the following theorem.

\section{Theorem 1.6}

$$
P_{n, N(M)}^{(k)}(x)=\sum_{j=0}^{n}\left(\begin{array}{l}
n \\
j
\end{array}\right) x^{n-j} P_{j, N(M)}^{(k)} .
$$

Hence, we arrive at the following definition.

Definition 1.7 Euler-type polynomials with order $k, P_{n, N(M)}^{(k)}$, are defined by means of the following generating functions:

$$
\mathcal{F}_{N(M)}^{(k)}(t, x)=\sum_{n=0}^{\infty} P_{n, N(M)}^{(k)}(x) \frac{t^{n}}{n !},
$$

where

$$
\left|t+\sum_{j=1}^{M} \frac{2 \pi i}{N_{j}}\right|<2 \pi .
$$

Note that there is one generating function for each value of $k$. These are given explicitly as follows:

$$
\begin{aligned}
\mathcal{F}_{N(M)}^{(k)}(t, x) & =\left(\frac{-1+\prod_{j=1}^{M} \xi\left(N_{j}\right)}{-1+e^{t} \prod_{j=1}^{M} \xi\left(N_{j}\right)}\right)^{k} e^{t x} \\
& =\sum_{n=0}^{\infty} P_{n, N(M)}^{(k)}(x) \frac{t^{n}}{n !} .
\end{aligned}
$$

We derive the following functional equation:

$$
\mathcal{F}_{N(M)}^{(k+l)}(t, x)=\mathcal{F}_{N(M)}^{(k)}(t, x) \mathcal{F}_{N(M)}^{(l)}(t)
$$


By using the above functional equation, we arrive at the following theorem.

\section{Theorem 1.8}

$$
P_{n, N(M)}^{(k+l)}(x)=\sum_{j=0}^{n}\left(\begin{array}{l}
n \\
j
\end{array}\right) P_{j, N(M)}^{(k)}(x) P_{n-j, N(M)}^{(l)}
$$

Proof By using (3), (6) and (7), we get

$$
\sum_{n=0}^{\infty} P_{n, N(M)}^{(k+l)}(x) \frac{t^{n}}{n !}=\sum_{n=0}^{\infty}\left(\sum_{j=0}^{n}\left(\begin{array}{l}
n \\
j
\end{array}\right) P_{j, N(M)}^{(k)}(x) P_{n-j, N(M)}^{(l)}\right) \frac{t^{n}}{n !} .
$$

By comparing the coefficients of $\frac{t^{n}}{n !}$ on both sides of the above equation, we get the desired result.

Substituting $x=0$ into (8), we obtain a convolution formula for the numbers by the following corollary.

\section{Corollary 1.9}

$$
P_{n, N(M)}^{(k+l)}=\sum_{j=0}^{n}\left(\begin{array}{l}
n \\
j
\end{array}\right) P_{j, N(M)}^{(k)} P_{n-j, N(M)}^{(l)} .
$$

By differentiating both sides of equation (2) with respect to the variable $x$, we obtain the following higher-order differential equation:

$$
\frac{\partial^{j}}{\partial x^{j}} \mathcal{F}_{N(M)}(t, x)=t^{j} \mathcal{F}_{N(M)}(t, x)
$$

Remark 1.10 Setting $N(M)=\left(N_{1}, 1,1, \ldots, 1\right), P_{n,\left(N_{1}, 1,1, \ldots, 1\right)}$ is reduced $P_{n, N_{1}}(x)(c f .[1])$. Therefore $P_{n, N}(x)$ was defined by generalized Bernoulli-Euler polynomials in [1] as follows:

$$
\sum_{n=0}^{\infty} P_{n, N}(x) \frac{t^{n}}{n !}= \begin{cases}\frac{t t^{t x}}{e^{t}-1}, & N=1 \\ \frac{\left(\xi_{N}-1\right) e^{t x}}{\xi_{N} e^{t}-1}, & N \geq 2\end{cases}
$$

so that, obviously,

$$
P_{n, 1}(x)=B_{n}(x)
$$

and

$$
P_{n, 2}(x)=E_{n}(x)
$$

Here $B_{n}(x)$ and $E_{n}(x)$ are Bernoulli polynomials and Euler polynomials, respectively (cf. $[1-19])$.

The Frobenius-Euler polynomial is defined as follows: 
Let $u$ be an algebraic number such that $1 \neq u \in \mathbb{C}$. Then the Frobenius-Euler polynomial $H_{n}(x, u)$ is defined by

$$
\frac{1-u}{e^{t}-u} e^{t x}=\sum_{n=0}^{\infty} H_{n}(x, u) \frac{t^{n}}{n !}
$$

where

$$
\left|t+\ln \frac{1}{u}\right|<2 \pi
$$

(cf. [1-19]).

Remark 1.11 Frobenius-Euler number is denoted by $H_{n}(u)$ such that $H_{n}(0, u)=H_{n}(u)$. Also, $H_{n}(x,-1)=E_{n}(x)(c f .[1-19])$.

By using Frobenius-Euler numbers, one can obtain the Frobenius-Euler polynomials as follows:

$$
H_{n}(x, u)=\sum_{j=0}^{n}\left(\begin{array}{l}
n \\
j
\end{array}\right) x^{n-j} H_{j}(u)
$$

(cf. $[1-19])$.

The Apostol-Bernoulli polynomial is defined as follows.

Definition 1.12 $[3,16]$ The Apostol-Bernoulli polynomial $\mathcal{B}_{n}(x, \lambda)$ is defined by

$$
\frac{t}{\lambda e^{t}-1} e^{t x}=\sum_{n=0}^{\infty} \mathcal{B}_{n}(x, \lambda) \frac{t^{n}}{n !}
$$

where $\lambda$ is the arbitrary real or complex parameter and

$$
|t|<|\ln \lambda| .
$$

Remark 1.13 For $\lambda=1$, we obtain that $\mathcal{B}_{n}(x, 1)=B_{n}(x)(c f$. [1-19]).

\section{A functional equation of generalized Euler-type polynomials}

Bayad, Aygunes and Simsek showed that for $a \equiv 1 \bmod (N)$, there exists a unique sequence of monic polynomials $\left(P_{n, N}\right)_{n \in \mathbb{N}_{0}}$ in $\mathbb{Q}\left(\xi_{N}\right)[x]$ with $\operatorname{deg} P_{n, N}=n$ such that

$$
T_{\chi_{a, N}}\left(P_{n, N}(x)\right)=a^{-n} P_{n, N}(x),
$$

where $a, N \in \mathbb{N}(c f .[1])$.

In this section, we give the following theorem.

Theorem 2.1 Let $a, N_{1}, N_{2}, \ldots, N_{M} \in \mathbb{N}$ and $a \equiv 1\left(\bmod N_{1} N_{2} \cdots N_{M}\right)$. Then there exists a sequence $\left(P_{n, N(M)}\right)_{n \in \mathbb{N}_{0}}$ in

$$
\mathbb{Q}\left(\xi\left(N_{1}\right) \xi\left(N_{2}\right) \cdots \xi\left(N_{M}\right)\right)[x]
$$


with

$$
\operatorname{deg} P_{n, N(M)}=n
$$

such that

$$
T_{\chi_{a, N(M)}}\left(P_{n, N(M)}(x)\right)=a^{-n} P_{n, N(M)}(x) .
$$

Proof Since $P_{n, N(M)} \in \mathbb{C}[x]$ and $T_{\chi_{a, N(M)}}: \mathbb{C}[x] \rightarrow \mathbb{C}[x]$, we get

$$
T_{\chi_{a, N(M)}}\left(P_{n, N(M)}(x)\right)=\sum_{k=0}^{a-1} \chi_{a, N(M)}(k) P_{n, N(M)}\left(\frac{x+k}{a}\right) .
$$

From the definition of $\chi_{a, N(M)}(k)$, we have

$$
T_{\chi_{a, N(M)}}\left(P_{n, N(M)}(x)\right)=\sum_{k=0}^{a-1}\left(\prod_{j=1}^{M} e^{\frac{2 \pi i k}{N_{j}}}\right) P_{n, N(M)}\left(\frac{x+k}{a}\right) .
$$

By using the generating function of $P_{n, N(M)}(x)$, we get

$$
\begin{aligned}
\sum_{n=0}^{\infty} & \sum_{k=0}^{a-1}\left(\prod_{j=1}^{M} e^{\frac{2 \pi i k}{N_{j}}}\right) P_{n, N(M)}\left(\frac{x+k}{a}\right) \frac{t^{n}}{n !} \\
= & \sum_{k=0}^{a-1}\left(\prod_{j=1}^{M} e^{\frac{2 \pi i k}{N_{j}}}\right) \sum_{n=0}^{\infty} P_{n, N(M)}\left(\frac{x+k}{a}\right) \frac{t^{n}}{n !} \\
= & \sum_{k=0}^{a-1}\left(\prod_{j=1}^{M} e^{\frac{2 \pi i k}{N_{j}}}\right) \frac{\left(\left(\prod_{j=1}^{M} e^{\frac{2 \pi i}{N_{j}}}\right)-1\right) e^{t\left(\frac{x+k}{a}\right)}}{\left(\prod_{j=1}^{M} e^{\frac{2 \pi i}{N_{j}}}\right) e^{t}-1} \\
= & \frac{\left.\left(\prod_{j=1}^{M} e^{\frac{2 \pi i}{N_{j}}}\right)-1\right) e^{\frac{t x}{a}}}{\left(\prod_{j=1}^{M} e^{\frac{2 \pi i}{N_{j}}}\right) e^{t}-1} \sum_{k=0}^{a-1}\left(\exp \left(\sum_{j=1}^{M} e^{\frac{2 \pi i k}{N_{j}}}\right)\right) \exp \left(\frac{t k}{a}\right) \\
= & \frac{\left(\left(\prod_{j=1}^{M} e^{\frac{2 \pi i}{N_{j}}}\right)-1\right) e^{\frac{t x}{a}}}{\left(\prod_{j=1}^{M} e^{\frac{2 \pi i}{N_{j}}}\right) e^{t}-1} \sum_{k=0}^{a-1}\left(\exp \left(\frac{t}{a}+\sum_{j=1}^{M} \frac{2 \pi i}{N_{j}}\right)\right)^{k} \\
= & \frac{\left(\left(\prod_{j=1}^{M} e^{\frac{2 \pi i}{N_{j}}}\right)-1\right) e^{\frac{t x}{a}}}{e^{t}\left(\exp \left(\sum_{j=1}^{M} \frac{2 \pi i}{N_{j}}\right)\right)^{a}-1} \\
\left(\prod_{j=1}^{M} e^{\frac{2 \pi i}{N_{j}}}\right) e^{t}-1 & e^{\frac{t}{a}\left(\exp \left(\sum_{j=1}^{M} \frac{2 \pi i}{N_{j}}\right)\right)-1} .
\end{aligned}
$$

Since $a \equiv 1\left(\bmod N_{1} N_{2} \cdots N_{M}\right)$, the following relation holds:

$$
\left(\exp \left(\sum_{j=1}^{M} \frac{2 \pi i}{N_{j}}\right)\right)^{a}=\exp \left(\sum_{j=1}^{M} \frac{2 \pi i}{N_{j}}\right)=\prod_{j=1}^{M} e^{\frac{2 \pi i}{N_{j}}}
$$

Therefore, we have

$$
\sum_{n=0}^{\infty}\left(\sum_{k=0}^{a-1}\left(\prod_{j=1}^{M} e^{\frac{2 \pi i k}{N_{j}}}\right) P_{n, N(M)}\left(\frac{x+k}{a}\right)\right) \frac{t^{n}}{n !}=\sum_{n=0}^{\infty} a^{-n} P_{n, N(M)}(x) \frac{t^{n}}{n !} .
$$


By comparing the coefficients of $\frac{t^{n}}{n !}$ on both sides of the above equation, we get the desired result.

Remark 2.2 A different proof of (10) is given in [2]. If we take $N_{1} \geq 2$ and $N_{2}=N_{3}=\cdots=$ $N_{M}=1$, we have the following functional equation:

$$
T_{\chi a, N_{1}}\left(P_{n, N_{1}}(x)\right)=a^{-n} P_{n, N_{1}}(x)
$$

which is satisfied for generalized Bernoulli-Euler polynomials in [1].

\section{Some properties of generalized Euler-type polynomials}

In this section, we obtain some relations between generalized Euler-type polynomials, Apostol-Bernoulli polynomials and Frobenius-Euler polynomials. Also, we give a formula to obtain the generalized Euler-type polynomials.

Theorem 3.1 Let $n \in \mathbb{N}$. Then we have

$$
P_{n+1, N(M)}(x)=P_{n, N(M)}(x)+\frac{\prod_{j=1}^{M} \xi\left(N_{j}\right)}{1-\prod_{j=1}^{M} \xi\left(N_{j}\right)} \sum_{k=0}^{n}\left(\begin{array}{l}
n \\
k
\end{array}\right) P_{k, N(M)}^{(2)}(x)
$$

Proof By differentiating both sides of equation (2) with respect to the variable $t$, we have

$$
\begin{aligned}
\sum_{n=0}^{\infty} P_{n+1, N(M)}(x) \frac{t^{n}}{n !} & =\frac{\partial}{\partial t} \mathcal{F}_{N(M)}(t, x) \\
& =\mathcal{F}_{N(M)}(t, x)+\left(\frac{\prod_{j=1}^{M} \xi\left(N_{j}\right)}{1-\prod_{j=1}^{M} \xi\left(N_{j}\right)}\right) e^{t} e^{t x}\left(\mathcal{F}_{N(M)}(t)\right)^{2} \\
& =\sum_{n=0}^{\infty} P_{n, N(M)}(x) \frac{t^{n}}{n !}+\left(\frac{\prod_{j=1}^{M} \xi\left(N_{j}\right)}{1-\prod_{j=1}^{M} \xi\left(N_{j}\right)}\right) e^{t}\left(\sum_{n=0}^{\infty} P_{n, N(M)}^{(2)}(x) \frac{t^{n}}{n !}\right)
\end{aligned}
$$

Therefore, we obtain

$$
\sum_{n=0}^{\infty} P_{n+1, N(M)}(x) \frac{t^{n}}{n !}=\sum_{n=0}^{\infty}\left(P_{n, N(M)}(x)+\frac{\prod_{j=1}^{M} \xi\left(N_{j}\right)}{1-\prod_{j=1}^{M} \xi\left(N_{j}\right)} \sum_{k=0}^{n}\left(\begin{array}{l}
n \\
k
\end{array}\right) P_{k, N(M)}^{(2)}(x)\right) \frac{t^{n}}{n !}
$$

By comparing the coefficients of $\frac{t^{n}}{n !}$, we obtain the desired result.

In the following theorem, we give a relation between the polynomials $P_{n, N(M)}(x)$ and Frobenius-Euler polynomials.

Theorem 3.2 [2] Let $n \in \mathbb{N}_{0}$. Then we have

$$
P_{n, N(M)}(x)=H_{n}\left(x, \prod_{j=1}^{M} \frac{1}{\xi\left(N_{j}\right)}\right)
$$


Proof By using the generating function of $P_{n, N(M)}(x)$, we have

$$
\sum_{n=0}^{\infty} P_{n, N(M)}(x) \frac{t^{n}}{n !}=\sum_{n=0}^{\infty} H_{n}\left(x, \prod_{j=1}^{M} \frac{1}{\xi\left(N_{j}\right)}\right) \frac{t^{n}}{n !} .
$$

In the above equation, if we compare the coefficients of $\frac{t^{n}}{n !}$, we get the desired result.

In the following theorem, we give a relation between $P_{n, N(M)}(x)$ and Apostol-Bernoulli polynomials.

Theorem 3.3 [2] Let $n \in \mathbb{N}$. Then we have

$$
P_{n-1, N(M)}(x)=\left(\prod_{j=1}^{M} \xi\left(N_{j}\right)-1\right) \frac{1}{n} \mathcal{B}_{n}\left(x, \prod_{j=1}^{M} \xi\left(N_{j}\right)\right) .
$$

Proof We arrange the generating function of generalized Euler-type polynomials as follows:

$$
\sum_{n=1}^{\infty} P_{n-1, N(M)} \frac{t^{n-1}}{(n-1) !}=\frac{\prod_{j=1}^{M} \xi\left(N_{j}\right)-1}{e^{t} \prod_{j=1}^{M} \xi\left(N_{j}\right)-1} e^{x t}
$$

Therefore, we have

$$
\sum_{n=1}^{\infty} P_{n-1, N(M)} \frac{t^{n-1}}{(n-1) !}=\sum_{n=1}^{\infty}\left(\frac{1}{n}\left(\prod_{j=1}^{M} \xi\left(N_{j}\right)-1\right) \mathcal{B}_{n}\left(x, \prod_{j=1}^{M} \xi\left(N_{j}\right)\right)\right) \frac{t^{n-1}}{(n-1) !} .
$$

In the above equation, if we compare the coefficients of $\frac{t^{n-1}}{(n-1) !}$, we get the desired result.

In the following theorem, it is possible to find the generalized Euler-type polynomials.

Theorem 3.4 Let $n \in \mathbb{N}_{0}$. Then we have

$$
P_{n, N(M)}(x)=\sum_{j=0}^{n}\left(\begin{array}{l}
n \\
j
\end{array}\right) x^{n-j} P_{j, N(M)} .
$$

Proof of (11) is the same as that of (5), so we omit it [2].

$$
\begin{aligned}
& P_{1, N(M)}=\frac{1}{\chi_{a, N(M)}^{-1}-1}, \\
& P_{2, N(M)}=\frac{2}{\left(\chi_{a, N(M)}^{-1}-1\right)^{2}}+\frac{1}{\chi_{a, N(M)}^{-1}-1}, \\
& P_{3, N(M)}=\frac{6}{\left(\chi_{a, N(M)}^{-1}-1\right)^{3}}+\frac{6}{\left(\chi_{a, N(M)}^{-1}-1\right)^{2}}+\frac{1}{\chi_{a, N(M)}^{-1}-1}
\end{aligned}
$$


and

$$
P_{4, N(M)}=\frac{24}{\left(\chi_{a, N(M)}^{-1}-1\right)^{4}}+\frac{36}{\left(\chi_{a, N(M)}^{-1}-1\right)^{3}}+\frac{14}{\left(\chi_{a, N(M)}^{-1}-1\right)^{2}}+\frac{1}{\chi_{a, N(M)}^{-1}-1} .
$$

By using (11), we have the following list for the generalized Euler-type polynomials:

$$
\begin{aligned}
P_{0, N(M)}(x)= & 1 \\
P_{1, N(M)}(x)= & x+\frac{1}{\chi_{a, N(M)}^{-1}-1}, \\
P_{2, N(M)}(x)= & x^{2}+x\left(\frac{2}{\chi_{a, N(M)}^{-1}-1}\right)+\left(\frac{2}{\left(\chi_{a, N(M)}^{-1}-1\right)^{2}}+\frac{1}{\chi_{a, N(M)}^{-1}-1}\right), \\
P_{3, N(M)}(x)= & x^{3}+x^{2}\left(\frac{3}{\chi_{a, N(M)}^{-1}-1}\right)+x\left(\frac{6}{\left(\chi_{a, N(M)}^{-1}-1\right)^{2}}+\frac{3}{\chi_{a, N(M)}^{-1}-1}\right) \\
& +\left(\frac{6}{\left(\chi_{a, N(M)}^{-1}-1\right)^{3}}+\frac{1}{\left(\chi_{a, N(M)}^{-1}-1\right)^{2}}+\frac{1}{\chi_{a, N(M)}^{-1}-1}\right)
\end{aligned}
$$

and

$$
\begin{aligned}
P_{4, N(M)}(x)= & x^{4}+x^{3}\left(\frac{4}{\chi_{a, N(M)}^{-1}-1}\right)+x^{2}\left(\frac{12}{\left(\chi_{a, N(M)}^{-1}-1\right)^{2}}+\frac{6}{\chi_{a, N(M)}^{-1}-1}\right) \\
& +x\left(\frac{24}{\left(\chi_{a, N(M)}^{-1}-1\right)^{3}}+\frac{24}{\left(\chi_{a, N(M)}^{-1}-1\right)^{2}}+\frac{4}{\chi_{a, N(M)}^{-1}-1}\right) \\
& +\left(\frac{24}{\left(\chi_{a, N(M)}^{-1}-1\right)^{4}}+\frac{36}{\left(\chi_{a, N(M)}^{-1}-1\right)^{3}}+\frac{14}{\left(\chi_{a, N(M)}^{-1}-1\right)^{2}}+\frac{1}{\chi_{a, N(M)}^{-1}-1}\right) .
\end{aligned}
$$

\section{Competing interests}

The author declares that he has no competing interests.

\section{Author's contributions}

The author completed the paper himself. The author read and approved the final manuscript.

\section{Acknowledgements}

Dedicated to Professor Hari M Srivastava.

The present investigation was supported by the Scientific Research Project Administration of Akdeniz University.

\section{Received: 11 December 2012 Accepted: 6 February 2013 Published: 26 February 2013}

\section{References}

1. Bayad, A, Aygunes, AA, Simsek, Y: Hecke operators and generalized Bernoulli-Euler polynomials. J. Algebra Number Theory, Adv. Appl. 3, 111-122 (2010)

2. Aygunes, AA: Hecke type operators and their application (Hecke tipi operatörler ve uygulamaları). PhD thesis, November (2012)

3. Apostol, TM: On the Lerch zeta function. Pac. J. Math. 2, 161-167 (1951)

4. Carlitz, L: The product of two Eulerian polynomials. Math. Mag. 36, 37-41 (1963)

5. Kim, T: Identities involving Frobenius-Euler polynomials arising from non-linear differential equations. J. Number Theory 132, 2854-2865 (2012)

6. Kim, DS, Kim, T: Some new identities of Frobenius-Euler numbers and polynomials. J. Inequal. Appl. (2012). doi:10.1186/1029-242X-2012-307

7. Kim, T, Rim, SH, Simsek, Y, Kim, D: On the analogs of Bernoulli and Euler numbers, related identities and zeta and L-functions. J. Korean Math. Soc. 45, 435-453 (2008)

8. Luo, Q-M, Srivastava, HM: Some relationships between the Apostol-Bernoulli and Apostol-Euler polynomials. Comput. Math. Appl. 10, 631-642 (2005)

9. Ozden, H, Simsek, Y, Srivastava, HM: A unified presentation of the generating functions of the generalized Bernoulli, Euler and Genocchi polynomials. Comput. Math. Appl. 60, 2779-2787 (2010) 
10. Satoh, J: $q$-analogue of Riemann's $\zeta$-function and q-Euler numbers. J. Number Theory 31, 346-362 (1989)

11. Shiratani, K: On Euler numbers. Mem. Fac. Sci., Kyushu Univ. 27, 1-5 (1975)

12. Simsek, Y: $q$-analogue of the twisted I-series and q-twisted Euler numbers. J. Number Theory 110, 267-278 (2005)

13. Simsek, Y: Generating functions for generalized Stirling type numbers, array type polynomials, Eulerian type polynomials and their application (2011). arxiv:1111.3848v1

14. Simsek, Y: Generating functions for q-Apostol type Frobenius-Euler numbers and polynomials. Axioms 1, 395-403 (2012). doi:10.3390/axioms1030395

15. Simsek, Y, Bayad, A, Lokesha, V: q-Bernstein polynomials related to $q$-Frobenius-Euler polynomials, I-functions, and q-Stirling numbers. Math. Methods Appl. Sci. 35, 877-884 (2012)

16. Srivastava, HM: Some generalizations and basic (or $q^{-}$) extensions of the Bernoulli, Euler and Genocchi polynomials. Appl. Math. Inf. Sci. 5, 390-444 (2011)

17. Srivastava, HM, Choi, J: Zeta and q-Zeta Functions and Associated Series and Integrals. Elsevier, Amsterdam (2012)

18. Srivastava, HM, Kim, T, Simsek, Y: q-Bernoulli numbers and polynomials associated with multiple $q$-zeta functions and basic L-series. Russ. J. Math. Phys. 12, 241-268 (2005)

19. Tsumura, $\mathrm{H}$ : A note on $q$-analogues of the Dirichlet series and $q$-Bernoulli numbers. J. Number Theory 39, 251-256 (1991)

doi:10.1186/1687-1812-2013-40

Cite this article as: Aygunes: Higher-order Euler-type polynomials and their applications. Fixed Point Theory and Applications 2013 2013:40.

\section{Submit your manuscript to a SpringerOpen ${ }^{\ominus}$ journal and benefit from:}

- Convenient online submission

Rigorous peer review

- Immediate publication on acceptance

- Open access: articles freely available online

- High visibility within the field

- Retaining the copyright to your article 